\title{
Scalable office-based health care
}

\author{
Gabriel A. Koepp, Chinmay U. Manohar, Shelly K. McCrady-Spitzer, and James A. Levine \\ Endocrine Research Unit, Mayo Clinic, Rochester, Minnesota 55905
}

\begin{abstract}
The goal of healthcare is to provide high quality care at an affordable cost for its patients. However, the population it serves has changed dramatically since the popularization of hospitalbased healthcare. With available new technology, alternative healthcare delivery methods can be designed and tested.

This study examines Scalable Office Based Healthcare for Small Business, where healthcare is delivered to the office floor. This delivery was tested in 18 individuals at a small business in Minneapolis, Minnesota. The goal was to deliver modular healthcare and mitigate conditions such as diabetes, hyperlipidemia, obesity, sedentariness, and metabolic disease.
\end{abstract}

The modular healthcare system was welcomed by employees $-70 \%$ of those eligible enrolled. The findings showed that the modular healthcare deliverable was feasible and effective. The data demonstrated significant improvements in weight loss, fat loss, and blood variables for at risk participants.

This study leaves room for improvement and further innovation. Expansion to include offerings such as physicals, diabetes management, smoking cessation, and pre-natal treatment would improve its utility. Future studies could include testing the adaptability of delivery method, as it should adapt to reach rural and underserved populations.

\section{BACKGROUND}

A recurring theme during 2009 and 2010 healthcare reform forums was that delivery and cost need to be addressed. ${ }^{1}$ In addition, research suggests that organizations such as the United Stated Department of Veteran Affairs need to create new models of healthcare deployment to reach rural veterans. ${ }^{2}$ Likewise, it has become apparent that developing novel and adaptable methods of healthcare delivery would benefit from taking advantage of rapidly advancing mobile technologies and a changing vista of healthcare access to reach the entire population.

The most commonplace model for health care delivery is via a physician or health care professional based in a physical health delivery center (e.g. doctor's office). Here we examined whether it is feasible for prevention and chronic disease services to be delivered to where people work; in this case in a small business because The Patient Protection and Affordable Care Act (P.L. 111-148) specifies program initiatives for small businesses. Currently, a negative correlation exists between healthcare out-of-pocket premiums and households acquisition of employer-sponsored health insurance policies. ${ }^{3}$ The majority of the United States population is insured through an employer-sponsored policy, thus emphasizing the need to reduce premiums in this area.

Author for Correspondence: James A. Levine, M.D. Ph.D., Endocrine Research Unit, 5-194 Joseph, Mayo Clinic, 200 First Street, SW, Rochester, MN 55905, USA, Phone: (507) 284-7636; Fax (507) 255-4828, levine.james@ mayo.edu. 
The workplace presents a particularly valuable opportunity to administer healthcare deliverables such as weight loss, increased hours of activity, screening for risk variables, nutrition education, and other low cost health interventions for several reasons. A healthcare delivery method that operates in collaboration with employers has the opportunity to reach a high percentage of the overall population, as approximately $90 \%$ of Americans are employed (http://www.bls.gov/ accessed May 21, 2010). It has been well documented that a lack of healthcare access is a contributing factor in decreased health. ${ }^{4}$ By deploying health at the office, access to healthcare is increased, and thus overall health should increase.

Moreover, there is a positive correlation between access and screening rate, ${ }^{5}$ while screening rate and good health are also positively correlated. The average employed American spends approximately 8 hours a day at work (http://www.bls.gov/ accessed May 21, 2010).

Considering that the average American spends most waking-time at work, work is associated with sedentariness, and sedentariness is associated with obesity, ${ }^{6}$ an office based healthcare offering would separate the association between the workplace and ill health. Moreover, by deploying throughout a company there is an opportunity to create culture change and increase employee accountability for their health. Many companies already have standardized health screens for their employees; with a small amount of effort the health screen could easily be turned into a complete healthcare interaction with well trained staff and appropriate follow up. ${ }^{7}$ Finally, of the five most costly diseases in the U.S., three, heart disease, hypertension and diabetes, are preventable. In fact, most health care costs are associated with chronic preventable disease which is amenable to office based delivery of healthcare. ${ }^{8}$

\section{SUBJECTS}

18 healthy volunteers were recruited at a staffing company in Minneapolis, MN aged $39.1 \pm$ 7.0 years, BMI $25.5 \pm 4 . \mathrm{kg} / \mathrm{m}^{2}$, fat mass $22.2 \pm 11.4 \mathrm{~kg}$ (Table 1 ). Subjects with preexisting chronic conditions were required to obtain permission from their personal physician in order to participate. Subjects were excluded if they were pregnant or nursing, because pregnancy may cause unknown health outcomes and thus compromise the data. Subjects that left the company during the study were also excluded from participating as they could not complete the study requirements.

\section{STUDY DESIGN}

We deployed Scalable Office Based Healthcare (SOBHC) directly to the office space of the small business. The subjects were studied for 6 months. Outcomes were measured on the first and last day of the study.

\section{SOBHC Evaluative Testing Procedures}

Participants underwent a simple evaluative process. Screening included a Health Risk Assessment (HRA), blood draw, and body composition. This process used clinical grade methods and required approximately 20 minutes of the participants time away from their work desk. In addition to the screen, participants underwent resting and walking energy expenditure testing to determine metabolic rates, and were given activity monitors to provide feedback regarding their caloric output. All testing was performed on the office work floor.

Measurement of Health Risk-Health risk was assessed using a questionnaire devised at Mayo Clinic. Here, subjects were categorized in three risk categories (low, medium, and high).

Blood Sampling -A venous blood sample was collected at Month 0 and 6. 
Measurements of weight and body composition-The volunteer's body weight and body composition were measured at the beginning of baseline and on the last day of the study. Air displacement plethysmography was used ${ }^{9}$ (BodPod; Concord, CA). These measurements were performed by a certified BodPod technician under standardized conditions (empty bladder, without shoes and wearing consistent, light and form-fitting clothing). The BodPod was calibrated before each use.

\section{SOBHC Personal Healthcare Intervention}

We used a three tiered health care delivery system. The first tier consisted of SOBHC screening that takes place on the office floor that was developed to work around employee workflow. The second tier used the combined skills of the following individuals: an exercise/activity counselor, a personal trainer, a dietitian, a nurse, and a diabetes management specialist. The third and final tier used the expertise of the staff physician and endocrinologist.

Those individuals identified as being, 'high risk' were recommended to change behavior based on the risk category. Participants with a BMI $>25 \mathrm{~kg} / \mathrm{m}^{2}$ were advised to lose weight and were given daily access to exercise and nutrition counselors, and the option to follow a weight loss program. Educational material was also offered for those individuals with poor nutrition/eating habits to train subjects how to make healthy food choices and prepare healthier food. High risk for lipid profiles were defined as having one or more of the following conditions: total cholesterol $>200 \mathrm{mg} / \mathrm{dL}$, low density lipids $>100 \mathrm{mg} / \mathrm{dL}$, triglycerides $>150 \mathrm{mg} / \mathrm{dL}$. Those individuals classified as having a high risk lipid profile were educated about the potential risks of their current state and on how to reduce these lipid levels. If the patient's total cholesterol $>250 \mathrm{mg} / \mathrm{dL}$, or Low Density Lipids $>150 \mathrm{mg} / \mathrm{dL}$, or Triglycerides $>300 \mathrm{mg} / \mathrm{dL}$ they were advised to see their personal physician. Those with High Density Lipids $<40 \mathrm{mg} / \mathrm{dL}$ were educated on how to increase HDL. Individuals with blood fasting blood Glucose $>100 \mathrm{mg} / \mathrm{dl}$ were monitored for 10 days and advised to see their physician if their blood glucose was consistently elevated. Individuals with a desire to increase muscle mass or attain a higher level of cardiovascular fitness were referred to the Personal Trainer.

In addition to the three tiered system, physical activity was measured using Actical (Respironics, Eidenhoven, Netherlands). This is a waterproof tri-axial accelerometer (26 x $26 \times 10 \mathrm{~mm})$.

Subjects wore the activity monitoring and data collecting device on their hip, clipped to a waistband or attached to an elastic belt for all waking hours. Subjects had weekly meetings with an exercise counselor where the activity data was retrieved and reset and subjects received activity feedback from the counselor. This device was used as a tool to identify negative or positive patterns in the participants' daily routine such as being seated for long periods of time, not getting adequate sleep, or going for a walk.

\section{The Integration and Environmental Changes for SOBHC}

Workplace ergonomic evaluations were conducted for space considerations including cubical size, conference room size and use, stair ways, and walking areas. In addition, external opportunities to promote active/healthy behavior such as art museums, parks, skyways, etc. were utilized. Careful consideration took place with site managers to be minimally disruptive to workflow when implementing environmental changes.

With the collaboration of senior management, facilities managers, and architects it was determined that a shared work station system would integrate most efficiently into the space 
available and provide users with adequate opportunity to walk while working. Treadmill desks ${ }^{10}$ (Steelcase, Grand Rapids, Michigan) were installed with a ratio of treadmill desks to participants being, 1 desk to 4 participants. The treadmill desks were equipped with hydraulic table top height adjustment to accommodate any users' proper ergonomics easily and therefore an ideal solution for the shared workstation environment. In addition, the traditional office desks were re-engineered to allow for a walking meeting track that would be non-disruptive to workflow. A treadmill desks conference room was configured to accommodate walking meetings that required a higher level of privacy.

Data and statistical analyses-For each group, the delta was calculated by using the difference between the beginning and ending values for each category respectively. Each category was then tested for statistical significance using a paired two tailed t-test.

Significance was defined as $\mathrm{p}<0.05$. Data are reported as mean \pm standard deviation unless otherwise stated.

\section{FINDINGS}

The Scalable Office Based Healthcare deployment was feasible. Architectural and ergonomic redesign of the office floor plan also proved to be feasible. For example, subjects easily adapted their workspace for the personalized interventions, deployed walking tracks themselves and the treadmill desks were small enough and adaptable enough to fit into a variety of open spaces allowing for user access.

There were no concerns regarding the SOBHC from participants, other workers, support staff or management. The study team found implementation and integration to be uncomplicated. Subjects scheduled their own healthcare appointments using an anonymous electronic system to fit around their work schedule. All appointments, evaluations and interventions were conducted on the office floor during normal working hours. For this, the study team was allocated two small unused spaces which we adapted for these testing and therapeutic applications.

Of those eligible, we recruited $70 \%$ of the employee population. Of those employees that started the intervention, all finished.

\section{SOBHC Outcome Measures}

Considered as a whole group, changes in health risk for the 18 SOBHC subjects were beneficial (Table 2). We studied how eighteen healthy employees responded to this environment over six months; nine of whom wanted to lose weight and nine of whom wanted to remain at the same body weight. Weight decreased for the whole cohort from $1443 \mathrm{~kg}^{18 \text {-subjects }}$ to $1372 \mathrm{~kg}^{18 \text {-subjects }}(\mathrm{P}<0.001)$ and body fat (air displacement plethysmography) from $400 \mathrm{~kg}^{18 \text {-subjects }}$ to $335 \mathrm{~kg}^{18 \text {-subject }}(\mathrm{P}=0.004)$. Individual weight loss averaged $4.0 \mathrm{~kg}(\mathrm{P}<0.001)$ and $90 \%$ of this was as body fat. BMI for the 18 study subjects decreased from $25.5 \pm 4.0 \mathrm{~kg} / \mathrm{m}^{2}$ to $24.2 \pm 3.5 \mathrm{~kg} / \mathrm{m}^{2}$ ( $\left.\mathrm{P}=0.0001\right)$, while the average weight loss was $4.0 \mathrm{~kg}(80.2 \pm 18.6 \mathrm{~kg}$ to $76.2 \pm 16.8 \mathrm{~kg}, \mathrm{P}=0.0002)$, and fat mass decreased $3.6 \mathrm{~kg}$ $(22.2 \pm 11.4$ to $18.6 \pm 10.8, \mathrm{P}=0.0006)$; all of which are statistically significant. Triglyceride and cholesterol levels also improved as they declined from $110.4 \pm 79.0 \mathrm{mg} / \mathrm{dL}$ to $74.5 \pm$ $31.2 \mathrm{mg} / \mathrm{dL}(\mathrm{P}=0.06)$ and $180.5 \pm 32.4 \mathrm{mg} / \mathrm{dL}$ to $173.6 \pm 25.1 \mathrm{mg} / \mathrm{dL}(\mathrm{P}=0.07)$ respectively.

For overweight and obese subjects; weight loss averaged, $6.1 \mathrm{~kg}(92.7 \pm 14.9 \mathrm{~kg}$ to $86.5 \pm$ $13.6 \mathrm{~kg}, \mathrm{P}=0.0003)$ of which $5.4 \mathrm{~kg}$ was fat $(29.6 \pm 11.2$ to $24.2 \pm 12.1 \mathrm{~kg}, \mathrm{P}=0.002)$. Total cholesterol decreased significantly for this group, from $195.5 \pm 28.2 \mathrm{mg} / \mathrm{dL}$ to $180.7 \pm 20.5$ $\mathrm{mg} / \mathrm{dL}(\mathrm{P}=0.004)$. 
Although, some lean subjects lost weight and improved blood variables, the changes were not significant (Table 2).

No subject was instructed or coerced to lose weight. Of the 18 subjects 6 overweight/obese and 3 lean expressed the desire to lose weight, while 3 overweight/obese and 6 lean chose to maintain their weight.

Those employees who wanted to maintain their weight achieved weight stability ( $74 \pm 14 \mathrm{~kg}$ c.f. $73 \pm 13 \mathrm{~kg}$ ). For employees who wished to lose weight ( $87 \pm 21 \mathrm{~kg}$ ), weight loss averaged $7 \mathrm{~kg}(\mathrm{P}<0.001)$ with $88 \%$ of this as body fat. Fat loss ranged from 2.2 to $10.3 \mathrm{~kg}$ and body fat decreased from $31 \pm 7 \%$ to $26 \pm 10 \%$ ( $\mathrm{P}=0.003$ ). Total cholesterol and triglycerides decreased significantly also (204 \pm 23 to $189 \pm 16 \mathrm{mg} / \mathrm{dl} ; \mathrm{P}<0.05$ and $143 \pm 98$ to $73 \pm 21 \mathrm{mg} / \mathrm{dl} ; \mathrm{P}<0.05$ ).

Subjects that desired weight loss were more active (walking equivalent $>1 \mathrm{mph}$ ) than in the weight stable group $941 \pm 431$ hours active/ 6 months/subject versus $666 \pm 219$ hours active/ 6 months/subject $(\mathrm{P}=0.045)$ suggesting that day-long activity may be important for weight loss in this setting.

Although, health care costs could not be analyzed in these preliminary studies, the company's revenue increased $9.8 \%$ over the first three-months of the study and $6.1 \%$ in the second three months of the study compared to the previous year. The company recorded its highest revenue for any one month at the mid-point of the study. Thus, SOBHC may provide people at work with the opportunity to lose weight whilst preserving productivity.

\section{DISCUSSION}

This study demonstrated that it is both feasible and potentially effective to deliver healthcare on the office floor. Considering the current state of healthcare and the annual increases in health insurance premiums, healthcare is in need of new and perhaps non-traditional deployment options. Scalable office based health care (SOBHC) highlights such an option.

By implementing SOBHC in an office, there is an opportunity to create change in the culture of health care delivery. For example, prior work has shown that overweight and obese individuals are seated two hours more per day more than their lean counterparts and prolonged sitting is associated with health risk. ${ }^{11,12}$ Hence, using activity promotion and environmental design as the centerpiece for healthy culture change, we were encouraged to note that overweight and obese subjects significantly lost weight, had greater activity and improved lipid variables. In addition, it was encouraging that weight loss in this study was predominantly fat mass; lean mass being preserved. In fact, both in the group as a whole and in the overweight/obese group, fat-free mass increased post-intervention (Whole group, 72.9 \pm 9.0 to $76.0 \pm 9.7 \% ; \mathrm{P}=0.004$. Overweight/obese group, $68.6 \pm 6.9$ to $782.7 \pm 9.6 \%$; $\mathrm{P}=0.01$ ). In contrast, weight loss treatments such as gastric bypass and caloric restriction significantly reduce muscle mass ${ }^{13}$. Overall, these initial findings suggest that SOBHC may precipitate fat loss with preservation of muscle mass.

Employee health could be impacted by healthcare reform, with potentially substantial implications for both employer and employee. ${ }^{14}$ It is not known, importantly, whether improving health care availability will actually improve health. Although from one small business, the data from this study suggest that improved access to health care might be associated with improved health outcomes. If the results from this study were proven robust in other businesses and SOBHC could be effectively scaled, this approach could potentially benefit the health of many people. 
We did not perform a formal economic analysis - as we propose in future studies. However, the incidental economic variables here were positive. Moreover, return on investment data for 'wellness strategies' suggest a positive ROI ranging from, $\$ 3-\$ 6$ for each $\$ 1$ invested ${ }^{15 ; 16 ; 17}$ possible outcomes could include fewer sickness-associated days, decreased healthcare costs and lower medication costs. ${ }^{18 ; 19 ; 20 ; 21}$ Thus, an approach such as SOBHC could be revenue positive for a company. Larger studies will need to be conducted to determine this, deploying appropriate fiscal metrics.

Integration of SOBHC not only benefits access to health because the health care is deployed where the person works. Also, the quality of healthcare, we believe, is likely to improve because it is provided conveniently (time- and place- efficient) and in an improved temporal sequence; for example blood pressure can be evaluated daily if necessary by the medical team. ${ }^{22 ; 23}$

Preventable illnesses accounts for most of health care expenditure and obesity impacts most preventable illnesses. ${ }^{8}$ Of the five most costly diseases in the US, three; heart disease, hypertension and diabetes, are directly associated with obesity, for example. Thus SOBHC from a national perspective may benefit health beyond a corporate perspective. The fact that more people are screened for illness than would have been enables prevention to be more effectively and efficiently deployed. SOBHC also represents an opportunity for epidemiological studies in an effort to track the incidence of disease.

Another advantage of an approach such as SOBHC is that much of the work is performed by non-physician healthcare professionals such as a nurses, nurse practitioners, physician assistants, or dieticians. Also SOBHC lends itself to the utilization of scalable tools such as mobile phone-based platforms. ${ }^{24}$ Thus, SOBHC/patient is likely to be less expensive than even an efficient physician driven primary care facility.

In conclusion, scalable office based health care delivered directly to the work space is feasible for small businesses. It is likely that this approach could benefit health in a cost efficient manner.

\section{LIMITATIONS}

There are several limitations that we acknowledge. First, this was a study conducted in a single small business. It is recognized that this company may not be representative of small businesses as whole. Nonetheless, the study demonstrated that the SOBHC deployment is feasible and has the potential to show health benefit. Second, we did not perform a formal economic analysis. In future studies we shall. Nonetheless, the indirect economic indices suggested benefit. Third, this approach was deployed in a small business environment and with the complexity of large corporations; it may be less easy to deploy SOBHC there. Conversely, oftentimes larger corporations have greater resources and so implementing SOBHC in a small business was warranted. Not all the employees in the company we investigated were enrolled due to limited resources. Large studies hereafter are undoubtedly warranted. A smaller preliminary study such as this is the first step in building the justification for larger studies. Also, the number of participants was sufficiently small that Type II error could have impacted the data set for the number of variables we studied. Finally, the scalability of SOBHC is not proven - just suggested - by this deployment. Scaling-up will require infrastructure development and subsequent testing.

\section{Acknowledgments}

The authors would like to thank the Clinical Research Unit at Mayo Clinic for their support of the study. 
Funding/Support: The project described was supported by Grant Number 1 UL1 RR024150 from the National Center for Research Resources (NCRR), a component of the National Institutes of Health (NIH), and the NIH Roadmap for Medical Research. Its contents are solely the responsibility of the authors and do not necessarily represent the official view of NCRR or NIH.

\section{References}

1. Blakely S. Employers, workers, and the future of employment-based health benefits. EBRI Issue Brief. 2010; (339):1-23. [PubMed: 20349733]

2. Leroy Y, Bocquet B, Mamouni A. Non-invasive microwave radiometry thermometry. Physiol Meas. 1998; 19(2):127-48. [PubMed: 9626678]

3. Abraham JM, Feldman R. Taking up or turning down: new estimates of household demand for employer-sponsored health insurance. Inquiry. 2010; 47(1):17-32. [PubMed: 20464952]

4. Wallace AE, Lee R, Mackenzie TA, et al. A longitudinal analysis of rural and urban veterans' health-related quality of life. J Rural Health. 2010; 26(2):156-63. [PubMed: 20447002]

5. Allen JD, Barlow WE, Duncan RP, et al. NIH State-of-the-Science Conference Statement: Enhancing Use and Quality of Colorectal Cancer Screening. NIH Consens State Sci Statements. 2010; 27(1)

6. McCrady SK, Levine JA. Sedentariness at work: how much do we really sit? Obesity (Silver Spring). 2009; 17(11):2103-5. [PubMed: 19390526]

7. Graffy J, Grant J, Williams K, et al. More than measurement: practice team experiences of screening for type 2 diabetes. Fam Pract. 2010

8. Stanton, MW. [accessed on Febuary 1, 2010, 2006] The High Concentration of U.S. Health Care Expenditures. 2006. Available at: http://www.ahrq.gov/research/ria19/expendria.htm\#diff4

9. Aleman-Mateo H, Huerta RH, Esparza-Romero J, et al. Body composition by the four-compartment model: validity of the BOD POD for assessing body fat in Mexican elderly. Eur J Clin Nutr. 2007; 61(7):830-6. [PubMed: 17228350]

10. Thompson WG, Foster RC, Eide DS, Levine JA. Feasibility of a walking workstation to increase daily walking. Br J Sports Med. 2008; 42(3):225-8. discussion 28. [PubMed: 17717060]

11. Hamilton MT, Hamilton DG, Zderic TW. Exercise physiology versus inactivity physiology: an essential concept for understanding lipoprotein lipase regulation. Exerc Sport Sci Rev. 2004; 32(4):161-6. [PubMed: 15604935]

12. Levine JA, Lanningham-Foster LM, McCrady SK, et al. Interindividual variation in posture allocation: possible role in human obesity. Science. 2005; 307(5709):584-6. [PubMed: 15681386]

13. Tamboli RA, Hossain HA, Marks PA, et al. Body Composition and Energy Metabolism Following Roux-en-Y Gastric Bypass Surgery. Obesity (Silver Spring). 2010

14. Fronstin P, Ross MN. Addressing health care market reform through an insurance exchange: essential policy components, the public plan option, and other issues to consider. EBRI Issue Brief. 2009; (330):1-22.

15. Baicker K, Cutler D, Song Z. Workplace Wellness Programs Can Generate Savings. Health Aff (Millwood). 2010

16. Cutler RG, Davis BJ, Ingram DK, Roth GS. Plasma concentrations of glucose, insulin, and percent glycosylated hemoglobin are unaltered by food restriction in rhesus and squirrel monkeys. J Gerontol. 1992; 47(1):B9-12. [PubMed: 1730847]

17. Milani RV, Lavie CJ. Impact of worksite wellness intervention on cardiac risk factors and one-year health care costs. Am J Cardiol. 2009; 104(10):1389-92. [PubMed: 19892055]

18. Gielen B, Remacle A, Mertens R. Patterns of health care use and expenditure during the last 6 months of life in Belgium: Differences between age categories in cancer and non-cancer patients. Health Policy. 2010

19. Laaksonen M, Pitkaniemi J, Rahkonen O, Lahelma E. Work arrangements, physical working conditions, and psychosocial working conditions as risk factors for sickness absence: bayesian analysis of prospective data. Ann Epidemiol. 2010; 20(5):332-8. [PubMed: 20382333]

20. van Oostrom SH, van Mechelen W, Terluin B, et al. A workplace intervention for sick-listed employees with distress: results of a randomised controlled trial. Occup Environ Med. 2010 
21. von Lengerke T, John J, Mielck A. Excess direct medical costs of severe obesity by socioeconomic status in German adults. Psychosoc Med. 2010; 7:Doc01. [PubMed: 20421952]

22. Fiscella K, Franks P, Clancy CM. Skepticism toward medical care and health care utilization. Med Care. 1998; 36(2):180-9. [PubMed: 9475472]

23. Mechanic D, McAlpine DD, Rosenthal M. Are patients' office visits with physicians getting shorter? N Engl J Med. 2001; 344(3):198-204. [PubMed: 11172143]

24. LaPenna AM. Alternative" healthcare: access as a revenue source in a consumer-driven market. J Healthc Manag. 2010; 55(1):7-10. [PubMed: 20210069] 

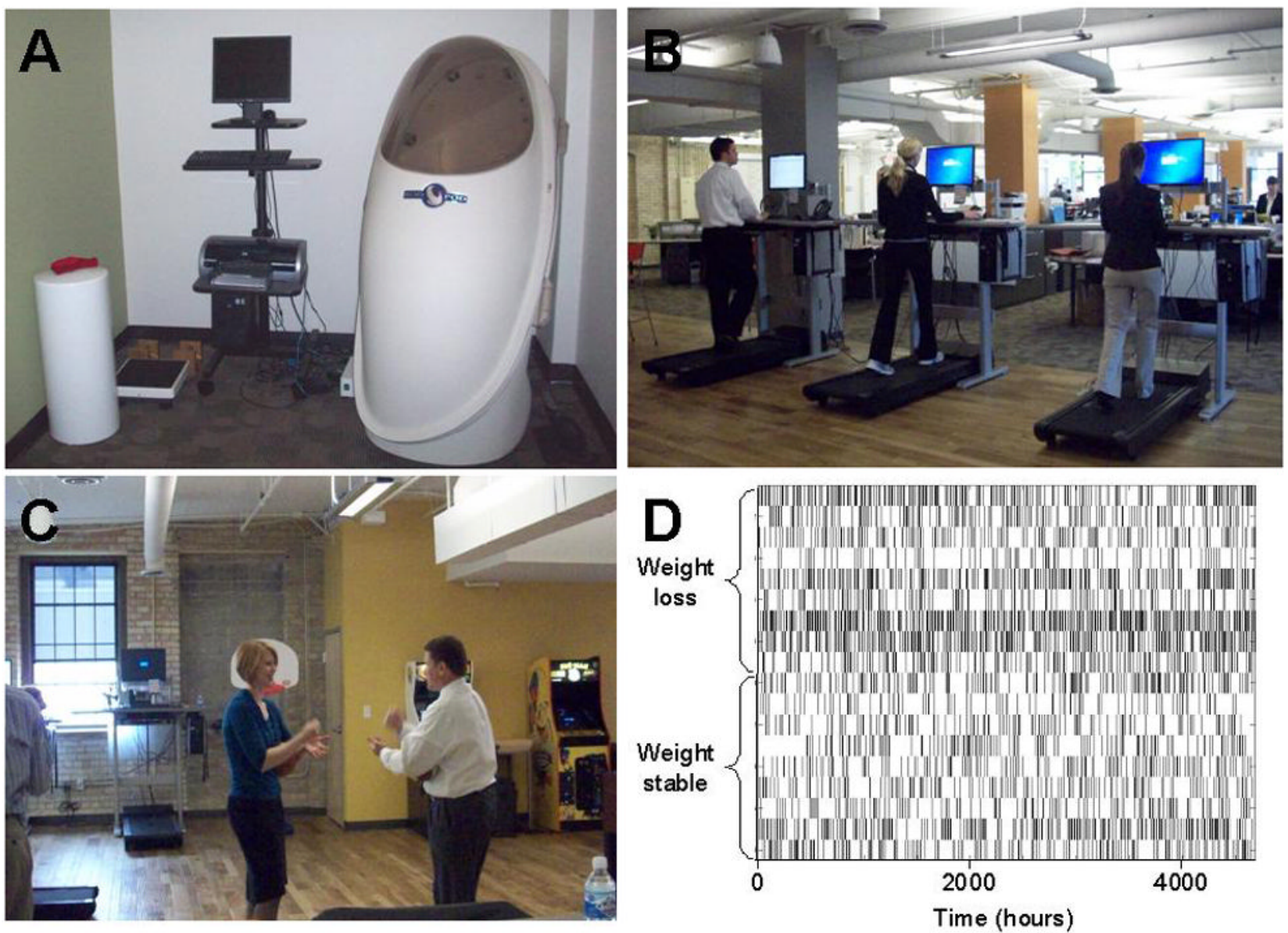

Figure 1. Scalable Office-based Health Care

(A) Mobile office health assessment laboratory (integrated into pre-existing office space). Integration of Health Environment; (B) Deployment of shared treadmill desks, (C) Managerial change to a standard meeting. (D) Bar-code system for demonstrating activity levels over the 6 month of the intervention for subjects electing to lose weight and those who chose to be weight stable. 


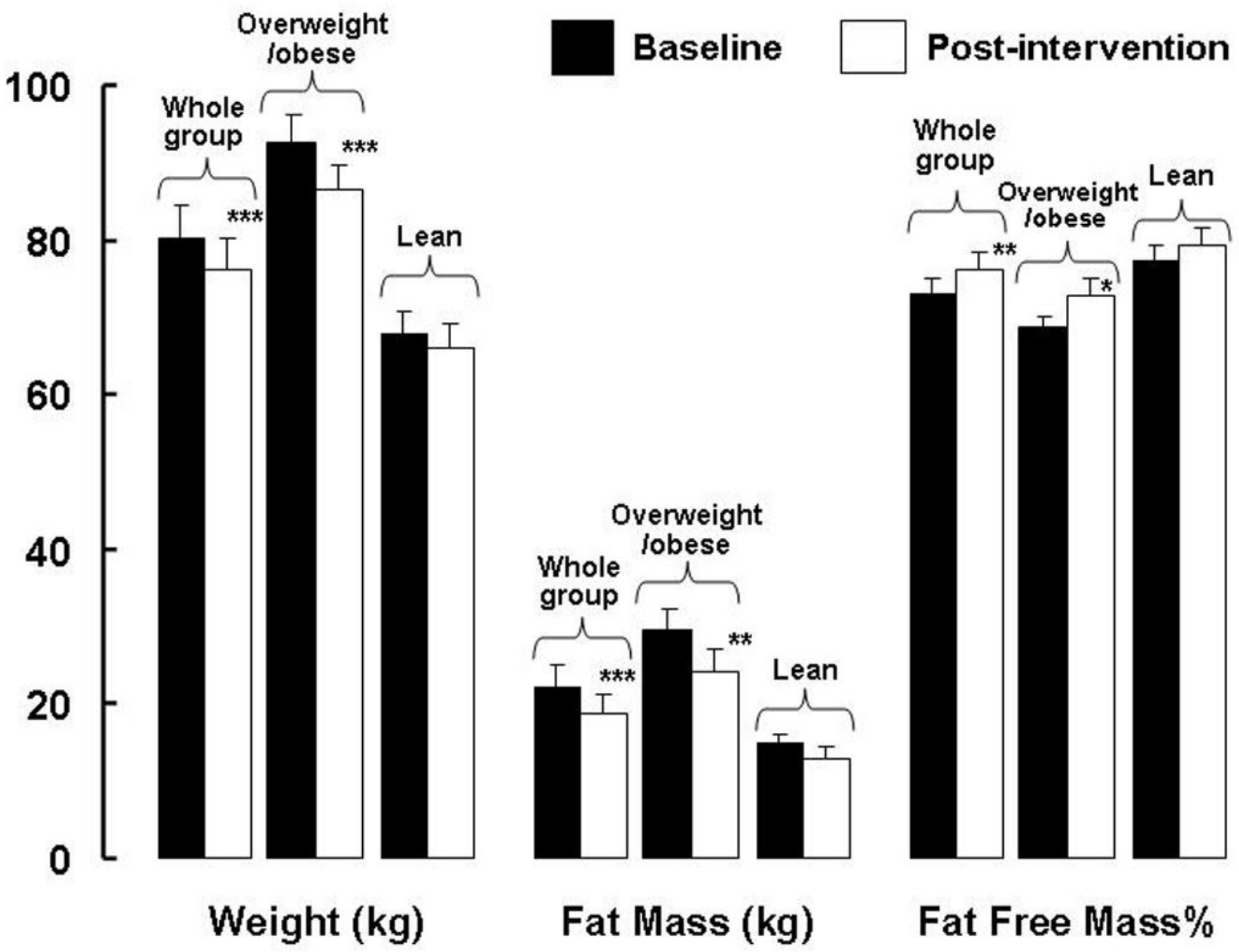

Figure 2. Body composition changes in 6 months Scalable Office-based Health Care in 18 subjects at baseline and after a 6 month intervention

Data are shown as mean \pm standard error of the mean. Pre-intervention versus postintervention, $* \mathrm{P}<0.01$, ** $\mathrm{P}<0.005$, *** $\mathrm{P}<0.001$. 


\section{Table 1}

Demographic details of 18 participants.

\begin{tabular}{|l|c|c|c|}
\hline & Whole Group & Over weight/obese & Lean \\
\hline Age (years) & $39.1 \pm 7.0$ & $39.1 \pm 6.2$ & $39.0 \pm 8.0$ \\
\hline Gender & $10 \mathrm{~F}, 8 \mathrm{M}$ & $3 \mathrm{~F}, 6 \mathrm{M}$ & $5 \mathrm{~F}, 2 \mathrm{M}$ \\
\hline Weight $(\mathrm{kg})$ & $80.2 \pm 18.6$ & $92.7 \pm 14.9$ & $67.7 \pm 12.7$ \\
\hline BMI $\left(\mathrm{kg} / \mathrm{m}^{2}\right)$ & $25.5 \pm 4.0$ & $28.4 \pm 3.4$ & $22.6 \pm 1.9$ \\
\hline
\end{tabular}

BMI, Body mass index. 


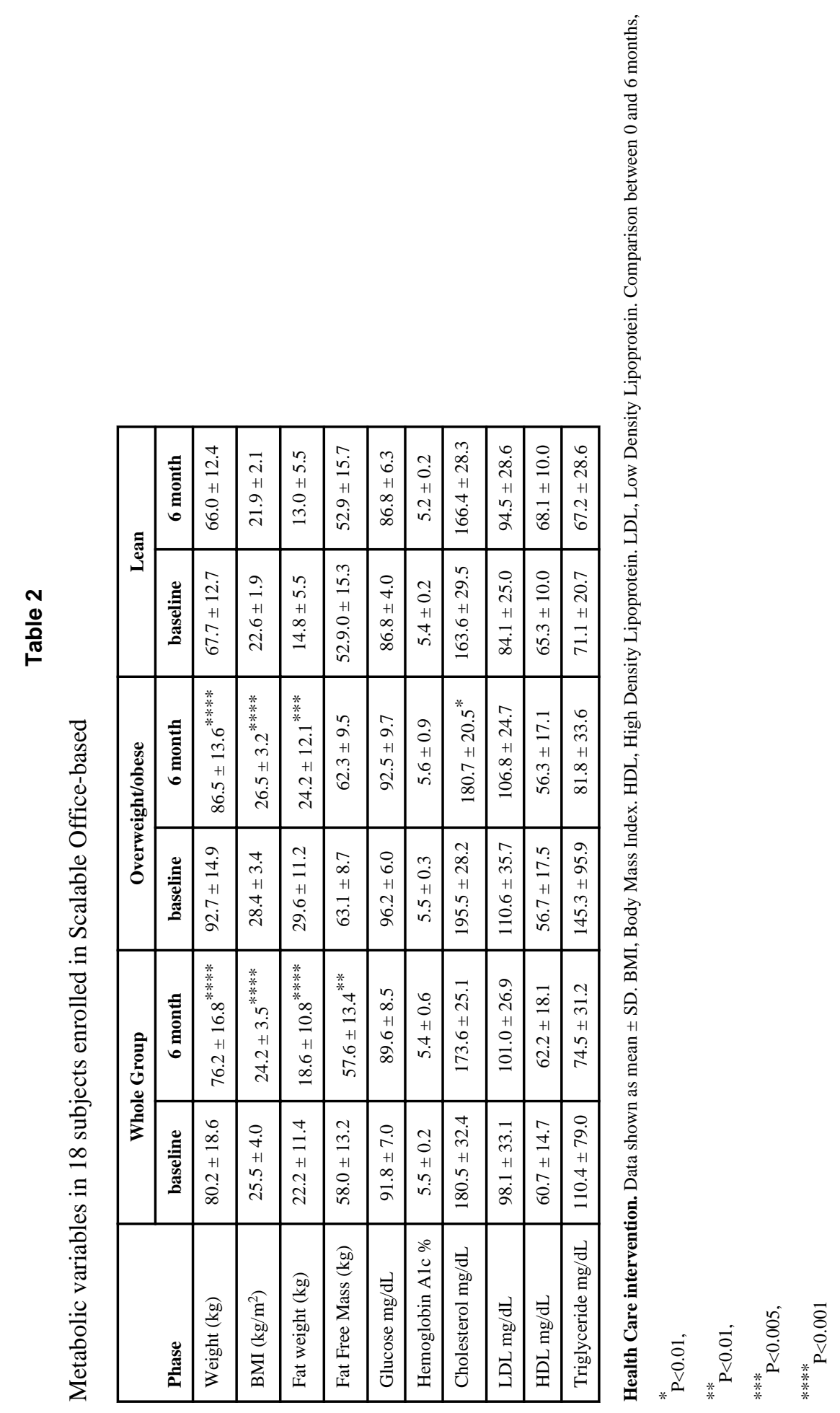

